\title{
Fast Watermarking of Traffic Images Secure Transmission in Effective Representation Mode
}

\author{
Ren Shuai $^{1, *}$, Lei Jingxiang ${ }^{1}$, Zhang Tao ${ }^{2}$ and Duan Zongtao ${ }^{1}$ \\ ${ }^{1}$ School of Information Engineering, Chang'an University, Xi' an 710064, China \\ ${ }^{2}$ School of Electronic and Control Engineering, Chang' an University, Xi'an 710064, China
}

Received: 27 Sep. 2013, Revised: 25 Dec. 2013, Accepted: 26 Dec. 2013

Published online: 1 Sep. 2014

\begin{abstract}
A static image secure transmission in effective representation mode for transportation systems was advanced based on fast watermarking theory. Firstly, the characteristics of image stream recognition in intelligent transportation system were analyzed and traffic image stream reorganization model was constructed. Then, according to effective representation mode, fast watermarking algorithms of image stream for intelligent transportation system were proposed, such as feature extraction-, compressive sensing- and sparse representation-based algorithms and detailed introduction of the first algorithm was given. Finally, referring to the evaluation criterion of digital watermarking and features of traffic static image, the performance of the above three algorithms are evaluated. The study shows that these three algorithms can satisfy different feature demands for traffic image secure transmission.
\end{abstract}

Keywords: traffic image stream, image security, fast watermarking, effective representation.

\section{Introduction}

In transport systems, traffic information acquisition is the primary task. Better than traditional detection methods such as ground loop, digital images can provide more intuitive and overall traffic information [1]. Digital image processing technology has been widely applied in ITS (intelligent transportation system) and generally can be classified as three application fields like visual-based traffic information pre-processing, traffic monitoring and intelligent vehicle navigation and traffic information storage and management. And traffic information storage and management is mainly used for inquiry of traffic accident and the target vehicles, storage and retrieval of traffic images and videos [2]. In ITS, there are lots of equipment generating massive amounts of visible information, which need to be transferred, stored and extracted as proofs and foundation. Meanwhile, technologies such as the new mobile communication, network, sensors, radio frequency identification devices (RFID) and cloud computing change the method of information access and release and gain obtain. Besides government, many subjects like enterprises can obtain massive traffic data from many channels. The commercial opportunities increase sharply but the data security will face a heavy lift. And it becomes the urgent issue of image security in ITS [3].

There are two types of visual data in ITS, such as static data (image) and dynamic data (video). In this paper, we study about the secure transmission of static image stream in ITS by watermarking. Because the static image stream is generated continuously, quickly and largely, the watermarking used should possess features such as real-time, rapidity and less computation [4]. We bring in fast watermarking to protect the static images in image stream. And the technical staff of public security bureau can distinguish the distortion and recover the essential features of the watermarked images even if they are processed by compression, sparse and some malicious tampering $[5,6]$.

\section{Image stream recognition system in effective representation mode}

The typical static image stream recognition system is shown in Figure 1.

In this system, the effective representation of images is the most important step. There are three types of effective representation in ITS: (1) Transform these data

\footnotetext{
*Corresponding author e-mail: maxwellren@qq.com
} 


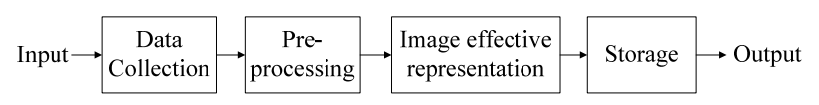

Fig. 1: Static image recognition system of ITS.

into several feature vectors with the optimal representative, shift, scale, rotation invariance; (2)Compress the massive static data to minimize redundancy; (3) Represent geometrical structures and energy of images with larger value and energy coefficient by sparse representation.

\section{Fast watermarking algorithm of traffic images}

Different from traditional watermarking, fast watermarking is of real-time performance in addition to robustness, invulnerability, invisibility and reversibility [7]Furthermore, search scopes for watermark embedding should be limited by specific methods, and also intensity and accuracy of robustness should be calculated.

Synchronous recording and expression are basic properties of static images in ITS. So fast watermarking applied in security authentication of static images for traffic system should satisfy instantaneity and fast realization. Now, studies on fast watermarking of static images are relatively rare, and fall into three categories such as regionalism theory-based (blocking and layering) [8,9], feature extraction theory-based (reversible comparison mapping and query table) [10] and convergence simplification theory-based (particle swarm, dynamic particle swarm, quantum theory, evolution theory and quantum evolution theory) [11].

\subsection{Fast watermarking for image stream}

Different from fast watermarking for image stream, many scholars present algorithms for video stream [12]. E. Vellasques et al presented the caption of image stream watermarking [11], and solved multi-layers duotone image stream watermarking used dynamic optimization. Then, E. Vellasques proposed an improved fast watermarking algorithm of image stream based on dynamic particle swarm optimization of Gaussian mixture model. By using HVS visibility threshold of wavelet domain, Wang Zhiwen et al [10] quantized wavelet coefficient of carrier image into quantum sequence with 0 and 1 and embed this sequence as a watermark. Then they found the best embedding location by QEA. Those methods were always used to locate and detect the position of embedded fast watermark.

Based on static image recognition in Fig.1, we present the stego-image stream recognition system in Fig. 2:

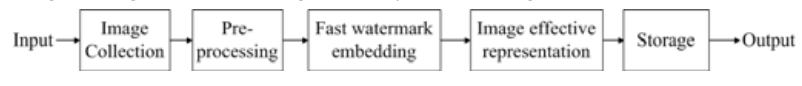

Fig. 2: Image recognition of image stream with fast watermarking.

Table 1: Robustness of fast watermarking algorithms based on different effective representations.

\begin{tabular}{c|c|c}
\hline $\begin{array}{c}\text { Effective } \\
\text { representation } \\
\text { methods }\end{array}$ & Characteristics & Robustness \\
\hline Feature extraction & Invariance & RTS transform \\
\hline $\begin{array}{c}\text { Compression } \\
\text { sensing }\end{array}$ & Vertical sampling layering & $\begin{array}{c}\text { JPEG, H.264, } \\
\text { Gaussian filtering }\end{array}$ \\
\cline { 2 - 2 } $\begin{array}{c}\text { Sparse } \\
\text { representation }\end{array}$ & Horizontal sampling layering & Multi-scale \\
\cline { 2 - 2 } & Multi-direction & $\begin{array}{c}\text { Scale and similarity } \\
\text { transform }\end{array}$ \\
\cline { 2 - 2 }
\end{tabular}

\subsection{Fast watermarking based on effective representation}

Data used for effective representation is of great energy and robustness. So we can embed watermark in effective representation data of image. Especially in traffic system, fragile label and robustness test value can be embedded in feature vector of image in order to be as the identification evidence. Using pre-processing algorithms based on energy and structure characteristics of image, static image can be divided into several sub-images. And these sub-images can be used as the fragile label and robustness test value embedding regions [13]. And effective representation methods can be used to pre-process static images and different effective representation methods can be used to improve the robustness of fast watermarking, shown as Table 1.

Effective representation of static image satisfies the energy structure pre-processing feature of carrier image. This advantage of effective representation can satisfy the maximum consistency between stego image and original image and invisibility.

\subsubsection{Fast watermarking based on feature extraction}

According to different features, there are several fast watermarking algorithms:

(1) Amplitude feature-based: using amplitude feature such as gray value of image pixel, three-color value, spectrum value and so on, which are basic features of image.

(2) Statistic feature-based: using histogram feature, statistic features (such as mean value, variance, energy and entropy), statistic features of pixel correlation description (such as self-correlation coefficient and covariance). 


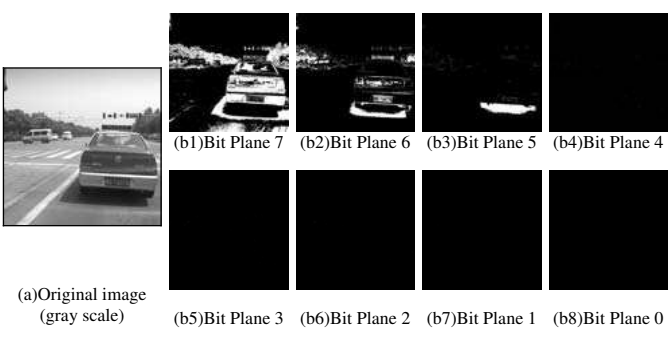

Fig. 3: Bit-plane decomposition of gray scale image.

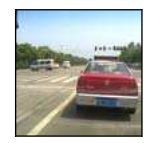
(a) Original image
(RGB)

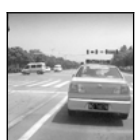

(b) Component $\mathrm{R}$

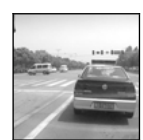

(c) Component G

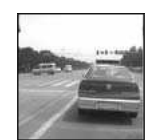

(d) Component B

Fig. 4: RGB color components.

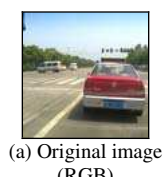

(RGB)

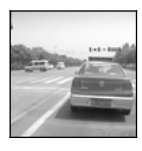

(b) Component $l$

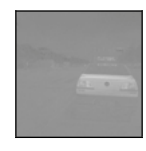

\begin{tabular}{ll} 
(c) Component $\alpha \quad$ (d) Component $\beta$ \\
\hline
\end{tabular}

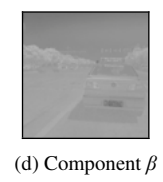

Fig. 5: Components of 1 color space.

(3) Geometric feature-based: using features such as area, perimeter, dispersity, elongation, gradient and curvature of curve, convexity-concavity and topology.

(4) Conversion coefficient feature-based: using Fourier transform coefficience, Hadamard transform, K-L transform, and other features such as texture and space structure of three dimensional models.

If the amplitude feature-based algorithms was used to embed fast watermark, bit plane theory (shown in Figure 3 ) and color space theory of digital image can be used in combination [17] .

RGB (Red Green Blue) is another resolution theory of image. Based on RGB color space, the RGB mode of traffic image in Figure 3 (a) was decomposed into three components in Figure 4.

1 color space is a color mode with larger space than RGB[?]. And the original image in Figure 4(a) can be decomposed into three components in Figure 5.

If choose statistical features, such as histogram, secret information can be embedded in low-gray scale and highgray scale region according to the secondary invisibility importance of those regions. The secret information can be embedded specifically by the modification of histogram [14]. Figure 6 shows the histogram of image in Figure 4 (a).

If choose geometrical features, such as gradient and curvature of curve, which can represent the structure of

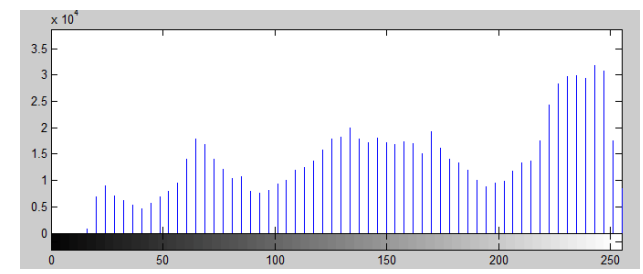

Fig. 6: Feature representation of histogram for static image.

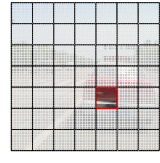

(a) Sub-region

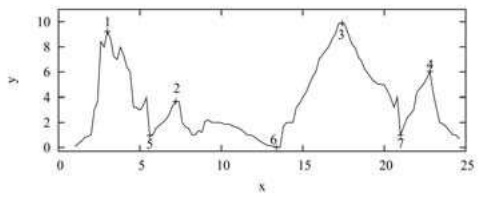

(b) Local height distribution of given sub-region (by Mean Shift)
Fig. 7: Feature representation of local height for static image.

static image, pixels importance of image can be sorted according to their curvature. This is consistent with the carrier structure feature analysis in information hiding preprocessing and can improve the invisibility. At the same time, we bring in local height theory as a new geometrical feature to describe the visibility importance of pixel or sub-region [15], shown as Figure 7. The pixels local height map of the sub-region with red border in Figure 7 (a) is shown in Figure 7 (b), in which 1, 2, 3 and 4 are local maximum points, and 5, 6 and 7 are local minimum points, others are background points.

If choose conversion coefficient feature, energy characteristics of static image can be used to embed the secret information with some common transform domain algorithms such as DCT, DFT, DWT, SVD, multi-scale, ridgelet transform and curve let transform. This is consistent with the carrier energy analysis in information hiding preprocessing and can improve the robustness and capacity. Especially, three transform domain algorithms such as multi-scale, ridgelet transform and curve let transform were barely used in watermarking although the recognition degree of them are high in image compression and sparse representation.

\subsubsection{Fast watermarking based on compression sensing}

Compression Sensing (CS) $[16,17]$ is based on new sampling theory, and can compress data while signal acquisition.

According to the maximum consistency principal, carrier images after CS pre-processing can be embedded watermarks faster, and the stego images are robust against common compression. 


\subsubsection{Fast watermarking based on sparse representation}

Based on traffic image features, the existing sparse representation algorithms are proposed around the study of compression issues of traffic images. Firstly, features of background and local similarity are studied. Then traffic images are sparse decomposed. According to the data distribution law, high-efficiency coding methods are studied. Generally, image sparse decomposition fast algorithm based on particle swarm was used to reduce the computation of image sparse decomposition.

\section{Conclusions}

In this paper, image stream recognition system and current fast watermarking algorithms of ITS were firstly studied. Then the effective representation mode of image stream recognition system was taken as the embedding node of fast watermarking. On account of huge data and real-time performance, three types of fast watermarking algorithms for image stream security authentication of ITS were proposed based on the previous study about watermarking. These three algorithms are respectively feature extraction-, compression sensing- and sparse representation-based, which are similar at the static image pre-processing step, so we just introduce the first algorithm in detail. And performance analysis indicates that those algorithms can improve the feature data security of static images in traffic system.

\section{Acknowledgments}

Our research was funded by two Projects: 1. Basic Research Project of Shaanxi Province Natural Science Foundation (Grant No. 2013JM8018). 2. The Special Fund for Basic Scientific Research of Central Colleges of Chang'an University (Grant No. 2013G1241118 and 2013G2241020).

\section{References}

[1] F. Y. Wang. IEEE Trans. Intell. Transport. Syst., 11, 630 (2010).

[2] X. F. Feng, J. Transport. Inform. Saf., 27, 84 (2009).

[3] N. Faouzi, H. Leung, A. Kurian, Inform. Fusion., 12, 4 (2011). W. J. Ma, S. R. Qu, J. Transport. Syst. Eng. Inform. Technol., 10, 49 (2010).

[4] M. Chen, F. Y. Wang, D. Zeng, IEEE Trans. Intell. Transport. Syst., 5, 329 (2004).

[5] X. Q. Miao, X. L. Jin, X. Y. Han, et al, J. Shanghai Jiao Tong Univ., 47, 253 (2013)

[6] X. H. Liu, Z. W. Kang, K. J. Wang, Appl. Res. Comput., 27, 2618 (2010).

[7] A. A. Mohammed, R. Minhas, Q.M. Jonathan Wu, M.A. Sid-Ahmed, Integr. Comput. Aided Eng., 17, 29 (2010).
[8] D. Coltuc, J. M.Chassery, IEEE Sig. Process. Lett., 14, 255 (2007).

[9] E. Vellasques, R. Sabourin, E. Granger, International Conference on Intelligent Information Hiding and Multimedia Signal Processing (IIH-MSP)., 139, (2010).

[10] Z. W. Wang, S. Z. Li, S. Z. Su, et al, J. Optoelectron. Laser., 21, 737 (2010).

[11] E. Vellasques, R. Sabourin, E. Granger, Appl. Soft Comput., 13, 3130 (2013).

[12] G. B. Yang, J. J. Li, Y. L. He, et al, AEU C Int. J. Electron. Commun., 65, 331 (2011).

[13] Chin-Chen Chang, Wei-Liang Tai ; Chia-Chen Lin, A Reversible Data Hiding Scheme Based on Side Match Vector Quantization, Circuits and Systems for Video Technology, IEEE Transactions on, 16, 1301-1308 (2006).

[14] S Ren, D Mu, T Zhang, W Hu, Study of information hiding algorithm based on GHM and color transfer theory, Optoelectronics Letters, Springer, 5, 454-458 (2009).

[15] J. J. Lin, D. H. Zhu, Y. B. Yang, J. Image Graphics, 16, 1841 (2011).

[16] D. L. Donoho, Y. Tsaig, Sig. Process., 86, 533 (2006).

[17] E. J. Candes, T. Tao, IEEE Trans. Inform. Theory., 52, 5406 (2006).

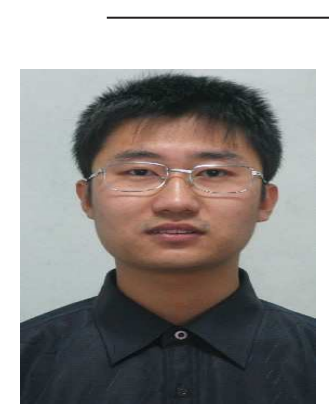

\section{Ren Shuai}

obtained his $\mathrm{PhD}$ from Northwestern Polytechnical University of China in 2009. $\mathrm{He}$ is a lecture in School of Information Engineering in Chang'an University. He has been engaged in Information hiding and Network security for 7 years. He published 23 scientific research articles in international publications and 2 are cited by SCI, 7 are cited by EI. He has carried out 5 tasks to study a plan in all, won patent 2 . During the last year he has written or co-edited for 5 textbooks.

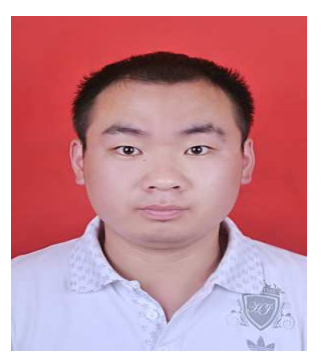




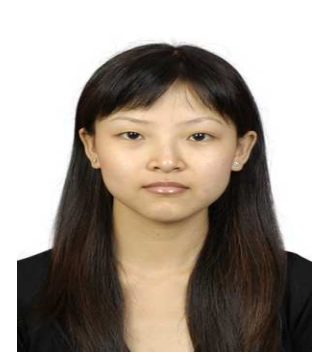

\section{Zhang Tao}

obtained her PhD from Northwestern Polytechnical University of China in 2012. She is a lecture in School of Electronic and Control Engineering in Changan University. She has been engaged in Information hiding and Network security for 8 years. She published 20 scientific research articles in international publications and 1 are cited by SCI, 6 are cited by EI. She has carried out 4 tasks to study a plan in all, won patent 1 .

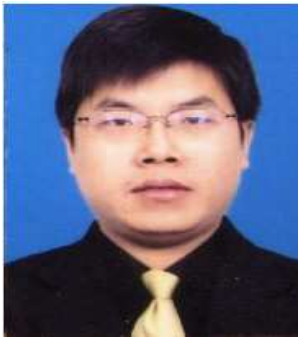

Duan Zongtao

received his Ph.D. from Northwestern Polytechnical University in 2006 . $\mathrm{He}$ is currently an associate professor in the School of Information Engineering at Changan University. His research interests include intelligent transportation system, cloud computing and big data processing. 